\title{
Supporting Information for 'Hydrophobic Core Repacking in a Coiled-Coil Dimer via Phage Display: Insights into Plasticity and Specificity at a Protein-Protein
}

\section{Interface'}

\author{
Jonathan R. Lai, ${ }^{\dagger}$ John D. Fisk, ${ }^{\dagger}$ Bernard Weisblum, ${ }^{+}$and Samuel H. Gellman ${ }^{*},+,, \xi$ \\ ${ }^{\dagger}$ Graduate Program in Biophysics, and Departments of ${ }^{\ddagger}$ Pharmacology and ${ }^{\S}$ Chemistry, University of \\ Wisconsin, Madison, Wisconsin 53706.
}

\section{SUPPLEMENTARY DATA}

Peptide Sequences.

Table S1 - Peptide Sequences

\begin{tabular}{|c|c|}
\hline Peptide & Sequence \\
\hline Base-p1 & Ac-AQLKKKLQALKKKNAQLKWKLQALKKKLAQ- $\mathrm{NH}_{2}$ \\
\hline Base-L21A & $\mathrm{AC}-\mathrm{AQLKKKLQALKKKNAQLKWKA} Q A L K K K L A Q-\mathrm{NH}_{2}$ \\
\hline Biotinylated Base-p1 & Biotin-SGSG-AQLKKKLQALKKKNAQLKWKLQALKKKLAQ- $\mathrm{NH}_{2}$ \\
\hline Biotinylated Base-L21A & Biotin-SGSG-AQLKKKLQALKKKNAQLKWKAQALKKKLAQ- $\mathrm{NH}_{2}$ \\
\hline Base-p1-SH & Ac-CGG-AQLKKKLQALKKKNAQLKWKLQALKKKLAQ-NH ${ }_{2}$ \\
\hline Base-L21A-SH & Ac-CGG-AQLKKKLQALKKKNAQLKWKAQALKKKLAQ- $\mathrm{NH}_{2}$ \\
\hline Acid-p1 & AC-AQLEKELQALEKENAQLEWELQALEKELAQ $-\mathrm{NH}_{2}$ \\
\hline Phage library ${ }^{a}$ & AQLEKELQALEKENA QXXEWXXXAXEKELAQ-SGSGS-pIII \\
\hline PLEIM & AC-AQLEKELQALEKENAQLEWEIQAMEKELAQ $-\mathrm{NH}_{2}$ \\
\hline Acid-p1-SH & AC-CGG-AQLEKELQALEKENAQLEWEL $Q A \mathbf{L} E K E L A Q-\mathrm{NH}_{2}$ \\
\hline PLEIM-SH & AC-CGG-AQLEKELQALEKENAQLEWEIQAMEKELAQ $-\mathrm{NH}_{2}$ \\
\hline
\end{tabular}

${ }^{\text {a }}$ Expressed as a fusion to pIII on M13 bacteriophage. 


\section{Construction and Panning of the Acid-p1 Phage Library.}

The Acid-p1-derived library sequences were expressed as fusions to pIII using the m663 M13 bacteriophage system $^{\mathrm{S} 1}$ via standard techniques. With four random positions, the theoretical library diversity of $1.6 \times 10^{5}$ sequences was well represented among our $2 \times 10^{6}$ transformants. Isolation and sequencing of over fifty random plaques confirmed that the Acid-p1-derived sequences had been inserted into the phage vector as desired, with variation at the appropriate positions (Table S2). A monoclonal phage displaying the Acid-p1 sequence was also isolated during library production and found to bind specifically to immobilized Base-p1 peptide by ELISA, indicating that these coded inserts were expressed and displayed properly on the surface of the phage particles.

Several rounds of affinity selection against biotinylated Base-L21A resulted in convergence on a single sequence: pLEIM (Table S2). Twenty-five binding clones were sequenced, 21 of which proved to be pLEIM. In contrast, panning against biotinylated Base-p1 resulted in a phage population containing many closely related sequences. The designed partner for Base-p1, Acid-p1 (residues at randomized positions: $\mathrm{L}_{17} \mathrm{E}_{20} \mathrm{~L}_{21} \mathrm{~L}_{24}$ ), was found twice in this pool, but the majority of the clones recovered from panning against Base-p1 contained the motif $\mathrm{L}_{17} \mathrm{X}_{20} \mathrm{I}_{21} \mathrm{Z}_{24}$, where $\mathrm{X}$ is predominantly polar residues and $\mathrm{Z}$ is hydrophobic. These results are consistent with host-guest studies that have shown Leu to be the most stabilizing residue at $\boldsymbol{d}$ positions of coiled-coil dimers, ${ }^{\mathrm{S} 2}$ whereas Ile is slightly preferred at $\boldsymbol{a}$ positions ${ }^{\mathrm{S} 3, \mathrm{~S} 4}$ (residues 17 and 24 are $\boldsymbol{d}$, and 21 is $\boldsymbol{a}$ ). The data imply that packing against the $\mathrm{Leu}_{21}$ side chain of Base-p1 provides only limited coiled-coil pairing specificity, since alternative hydrophobic side chains are acceptable at positions 21 and 24 of the opposing helix. In contrast, convergence on a single sequence when the library was panned against Base-L21A, suggests that Basep1-L21A would prefer to dimerize with pLEIM relative to very similar sequences (e.g., Acid-p1). 
Table S2 - Clones from Library Production and Panning

\begin{tabular}{|c|c|c|c|}
\hline Clone & \multicolumn{3}{|c|}{ Sequence } \\
\hline \multicolumn{4}{|c|}{ Representative clones isolated during library production ${ }^{a}$} \\
\hline Phage library & AQLEKELQALEKENAQXE & $W \times x Q$ & AXEKELAQ-SGSGS-PIII \\
\hline pSeq 1 & $\mathbf{Y}$ & RL & $\mathbf{v}$ \\
\hline pseq 2 & $Q$ & FG & $\mathbf{L}$ \\
\hline pSeq 3 & G & TS & $\mathbf{T}$ \\
\hline pseq 4 & I & VL & $\mathbf{L}$ \\
\hline pseq 5 & $\mathbf{T}$ & $\mathbf{F F}$ & $\mathbf{Q}$ \\
\hline pAcid-p1 & $\mathbf{L}$ & EL & $\mathbf{L}$ \\
\hline \multicolumn{4}{|c|}{ Clones from panning against Base-p1-L21A } \\
\hline PLEIM (21) & $\mathbf{L}$ & EI & $\mathbf{M}$ \\
\hline PLDIL (2) & $\mathbf{L}$ & DI & $\mathbf{L}$ \\
\hline pLELL [pACid-p1] (1) & $\mathbf{L}$ & EL & $\mathbf{L}$ \\
\hline PLLID (1) & $\mathbf{L}$ & LI & $\mathbf{D}$ \\
\hline \multicolumn{4}{|c|}{ Clones from panning against Base-p $1^{\mathrm{b}}$} \\
\hline PLEIL (4) & $\mathbf{L}$ & EI & $\mathbf{L}$ \\
\hline PLELL [pAcid-p1] (2) & $\mathbf{L}$ & EL & $\mathbf{L}$ \\
\hline PLSII (2) & $\mathbf{L}$ & SI & I \\
\hline PLMIY (2) & $\mathbf{L}$ & MI & $\mathbf{Y}$ \\
\hline PLNIL (2) & $\mathbf{L}$ & NI & $\mathbf{L}$ \\
\hline PLSLL (1) & L & SL & $\mathbf{L}$ \\
\hline PLAIL (1) & $\mathbf{L}$ & AI & $\mathbf{L}$ \\
\hline PLDIL (1) & $\mathbf{L}$ & DI & $\mathbf{L}$ \\
\hline PMLIW (1) & $\mathbf{M}$ & LI & w \\
\hline PLHII (1) & L & HI & I \\
\hline PIQIL (1) & I & QI & $\mathbf{L}$ \\
\hline pLDIV (1) & L & DI & $\mathbf{v}$ \\
\hline PMTIF (1) & $\mathbf{M}$ & TI & $\mathbf{F}$ \\
\hline PLTIY (1) & L & TI & $\mathbf{Y}$ \\
\hline PLDIA (1) & L & DI & $\mathbf{A}$ \\
\hline PMQLW (1) & $\mathbf{M}$ & QL & $\mathbf{W}$ \\
\hline PMMIY (1) & $\mathbf{M}$ & MI & $\mathbf{Y}$ \\
\hline PLDLL (1) & $\mathbf{L}$ & DL & $\mathbf{L}$ \\
\hline Consensus $^{c}$ (16) & L & $\mathrm{XI}$ & $\mathbf{z}$ \\
\hline
\end{tabular}

${ }^{a}$ Over fifty random plaques were isolated and sequenced during library construction; five representative sequences are shown here. All library clones contained the desired insert with variation at the expected positions. The clone diplaying the Acid-p1 sequence (pAcid-p1) was also isolated and found to bind specifically to immobilized Base-p1, indicating that the coding insert is expressed in a functional form on the surface of the phage. ${ }^{b}$ Twenty-five binding clones were sequenced after 4 rounds of selection against each peptide. The number of times each sequence was found is indicated in parentheses. ${ }^{c} \mathrm{X}$ is any amino acid, but predominantly polar residues, and $\mathrm{Z}$ is any hydrophobic residue. 


\section{Thermal Denaturations.}

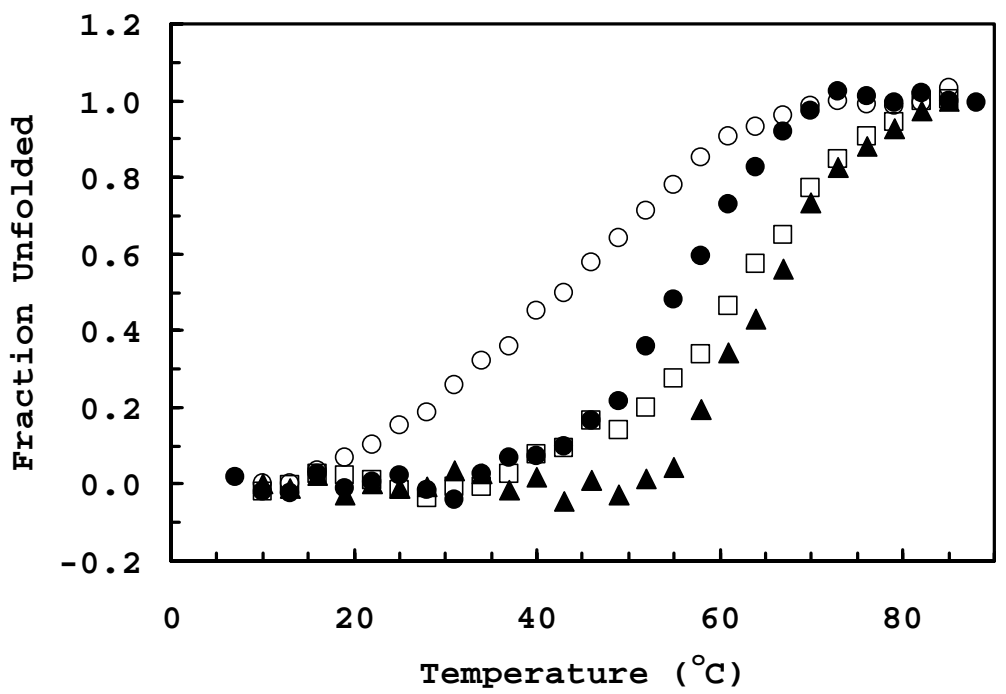

Figure S1. Thermal denaturation data for Base-L21A/pLEIM (•), Base-L21A/Acid-p1 (o), Basep1/Acid-p1 ( $\mathbf{\Delta}$ ), and Base-p1/pLEIM ( $\square$ ) at $10 \mu \mathrm{M}$ total peptide concentration in phosphate-buffered saline (PBS, $10 \mathrm{mM}$ phosphate, $150 \mathrm{mM} \mathrm{NaCl}$ ), $\mathrm{pH}$ 7.0.

\section{Urea Denaturations.}
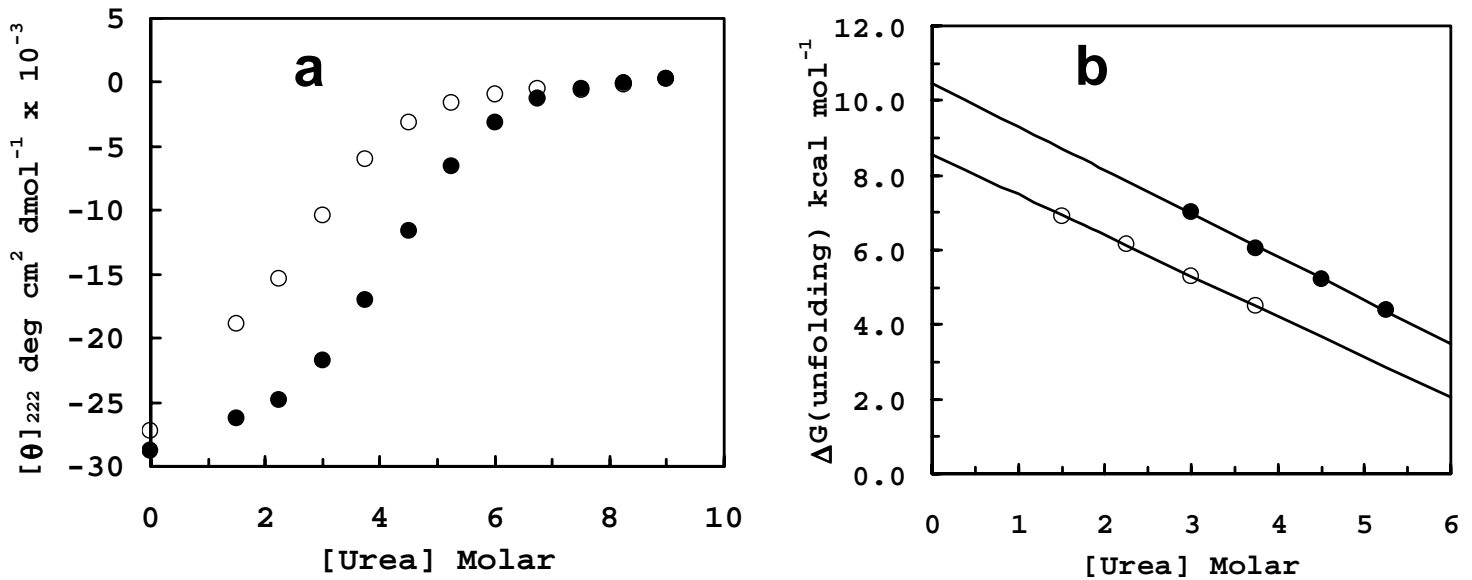

Figure S2. (a) Urea denaturation of Base-L21A/pLEIM (•) and Base-L21A/Acid-p1 (o) in PBS, pH 7.0 at $25{ }^{\circ} \mathrm{C}$. (b) Extrapolation to zero denaturant give $\Delta \mathrm{G}_{\mathrm{unf}}$ of $10.5 \mathrm{kcal} / \mathrm{mol}$ for Base-L21A/pLEIM and $8.6 \mathrm{kcal} / \mathrm{mol}$ for Base-L21A/Acid-p1. The uncertainty of $\Delta \mathrm{G}_{\text {unf }}$ values is $\pm 5 \%$. 


\section{Analytical Ultracentrifugation.}
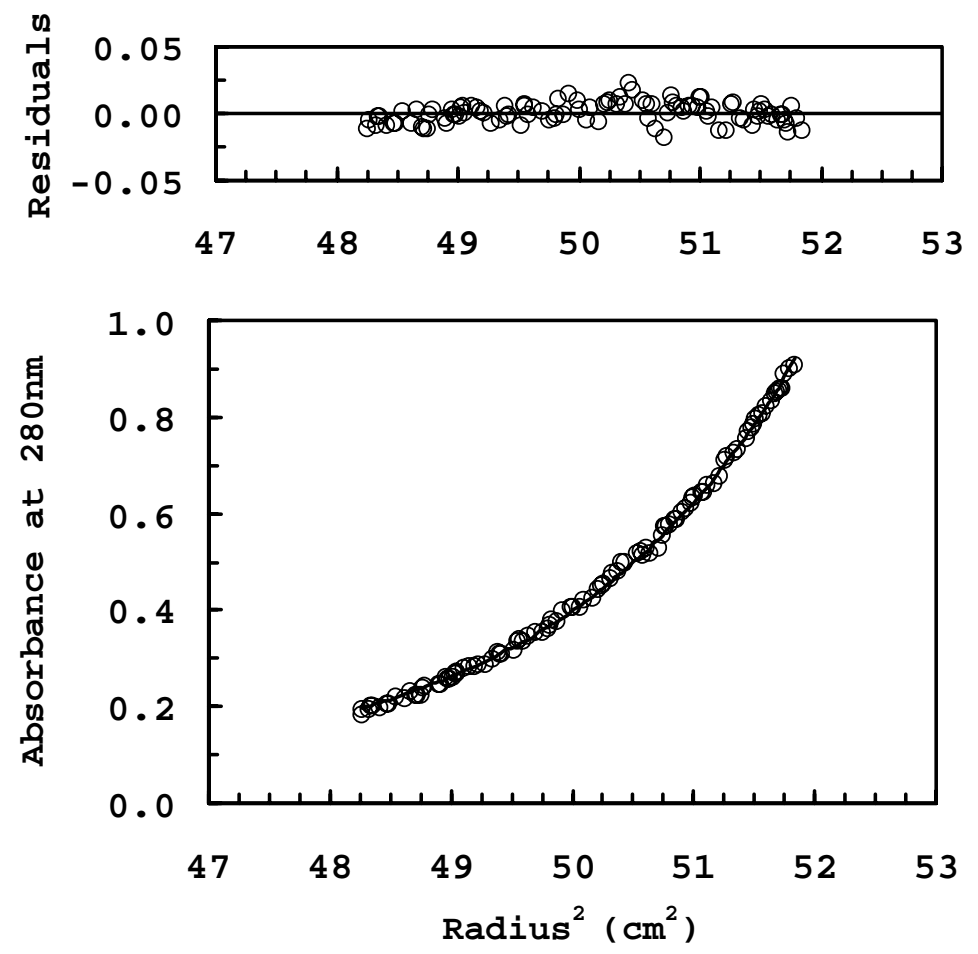

Figure S3. Representative analytical ultracentrifugation data for Base-L21A/pLEIM at $80 \mu \mathrm{M}$ total peptide concentration and $36 \mathrm{krpm}$. The data fit well to an ideal single species model of molecular weight $7130 \mathrm{~g} / \mathrm{mol}$. The expected mass of the Base-p1-L21A/pLEIM heterodimer is $7098 \mathrm{~g} / \mathrm{mol}$. 


\section{Thiol Exchange Assay.}

a
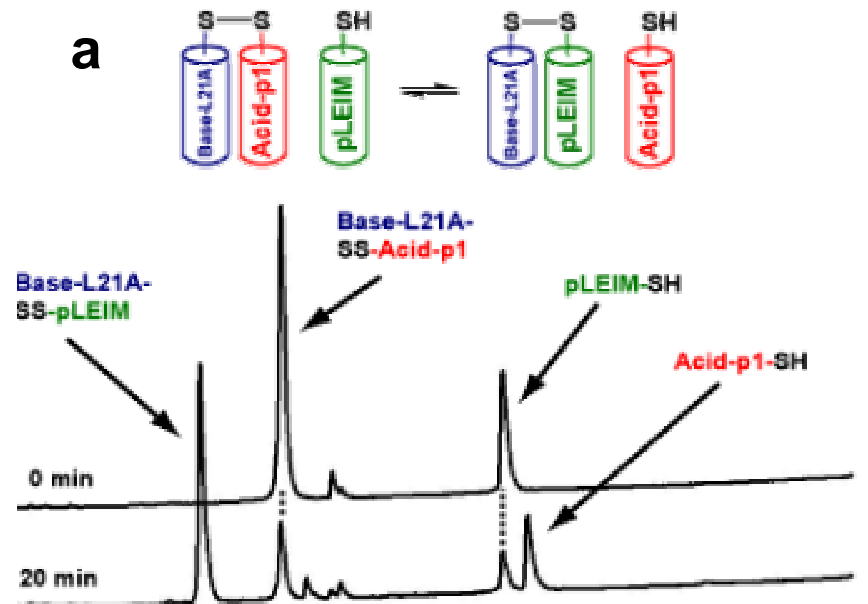
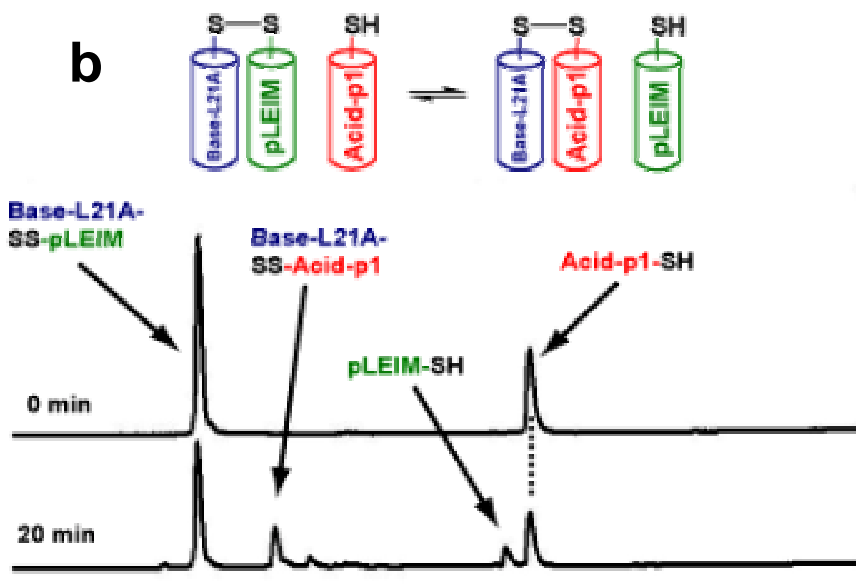

Figure S4. (a) Thiol exchange assay as monitored on a C4 analytical column by RP-HPLC beginning with the Base-L21A-SS-Acid-p1 dimer. Disulfide rearrangement produces the Base-L21A-SS-pLEIM dimer and free Acid-p1-SH. At equilibrium, the phage-derived Base-L21A-SS-pLEIM dimer is preferred over Base-L21A-SS-Acid-p1 by $\sim 3: 1$. The assay was performed at room temperature in PBS (pH 7.0) containing $1 \mathrm{mM}$ EDTA. The concentration of each peptide was $20 \mu \mathrm{M}$. Several independent trials yielded identical results. (b) Thiol exchange assay performed in the opposite direction with BaseL21A-SS-pLEIM and Acid-p1-SH as the starting species. Disulfide rearrangement again results in a $\sim 3: 1$ ratio of Base-L21A-SS-pLEIM to Base-L21A-SS-Acid-p1.

\section{METHODS}

Phage Display. General techniques were as described by Kay et al. ${ }^{\mathrm{S} 5}$ Library DNA was assembled by cloning a $118 \mathrm{bp}$ insert corresponding to the amino acid sequence AQLEKELQALEKENAQ్EW $\underline{\mathbf{X}}$ $\underline{\mathbf{X}} Q A \underline{\mathbf{X}} E K E L A Q G S G S G$ (where $\underline{\mathbf{X}}$ is any amino acid, encoded by the random codon $N N B$ where $\mathrm{N}=$ $\mathrm{A} / \mathrm{T} / \mathrm{G} / \mathrm{C}$ and $\mathrm{B}=\mathrm{T} / \mathrm{C} / \mathrm{G})$ upstream of the $p I I I$ gene in the m663 phage vector system. ${ }^{\mathrm{S} 1}$ Construction of the library was then achieved by transformation of the DNA into electrocompetent $E$. coli JS5 cells (Bio-Rad) and isolation of the resulting phage. Panning was performed using a modified protocol from Kay et al. ${ }^{\mathrm{S5,S6}}$ Briefly, wells in Costar high-binding EIA/RIA 96-well plates were coated with 
streptavidin $(500 \mathrm{ng} /$ well in $100 \mathrm{mM} \mathrm{NaHCO}$, $\mathrm{pH} \mathrm{8.5)} \mathrm{for} 1-2 \mathrm{hr}$ at room temperature, followed by treatment with Pierce blocking solution at $4{ }^{\circ} \mathrm{C}$ overnight. Biotinylated peptides were added (150 ng/well) in PBS containing $0.1 \%$ Tween 20 (PBS-Tween) and allowed to bind for $1-3 \mathrm{hr}$. Unbound streptavidin sites were then blocked for $20 \mathrm{~min}$ by adding $25 \mu \mathrm{L}$ of a saturated biotin solution. The wells were rinsed with PBS-Tween, phage solutions were added, and the plates were incubated at room temperature for $3-4 \mathrm{hr}$. Following phage binding, the wells were rinsed with wash solution (the wash solution was PBS-Tween for rounds $1-3$, and PBS containing 1.5 M urea for round 4), and then binding phage were eluted with $50 \mathrm{mM}$ glycine- $\mathrm{HCl}, \mathrm{pH} 2.0$ (incubated 25 min then neutralized with $200 \mathrm{mM}$ phosphate, $\mathrm{pH}$ 7.5). Amplification of the resulting phage population was achieved by infection of XL-1 Blue E. coli (Stratagene) at $37{ }^{\circ} \mathrm{C}$ for $6-12 \mathrm{hr}$. This process (a round of selection) was repeated three more times (for a total of four rounds of selection). Individual clones were isolated by plating out eluted phage, and phage binding was confirmed by ELISA. Sequencing was performed at the University of Wisconsin Biotechnology Center Sequencing Facility.

Peptide Synthesis and Purification. All peptides were synthesized using standard Fmoc chemistry with 2-(1H-Benzotriazole-1-yl)-1,1,3,3-tetramethyluronium hexafluorophosphate $\quad$ (HBTU)/NHydroxybenzotriazole (HOBt) activation on either an Applied Biosystems Model 432A Synergy automated peptide synthesizer or an Applied Biosystems Pioneer system. An aminomethylfunctionalized (AM) Rink amide polystyrene resin (Applied Biosystems) was employed with N- $\alpha$-Fmoc amino acids (with appropriate orthogonal sidechain protecting groups) purchased from Novabiochem. Capping of peptide N-terminal amino groups was achieved by treatment with coupling reagents and glacial acetic acid or biotin. After all residues were introduced, simultaneous cleavage from resin and sidechain deprotection was achieved by treatment of the resin with a trifluoroacetic acid (TFA)/1,2ethanedithiol (EDT)/water $(95 / 2.5 / 2.5 \mathrm{v} / \mathrm{v} / \mathrm{v})$ cocktail for $4-5 \mathrm{hr}$. Following removal of the resin, crude peptides were precipitated in cold diethyl ether (and the pellet washed with $2 \times 50 \mathrm{~mL}$ of cold diethyl 
ether), dissolved in acetonitrile/water, and lyophilized. Crude peptides were purified by reverse-phase high-pressure liquid chromatography (RP-HPLC) on a Vydac C4 silica column $(8 \mu \mathrm{m}, 250 \mathrm{~mm}$ x 25 $\mathrm{mm}$ ) using a non-linear gradient of water/acetonitrile containing $0.1 \%(\mathrm{v} / \mathrm{v})$ TFA. Peptide identity was confirmed by matrix-assisted laser desorption/ionization time-of-flight spectrometry (MALDI-TOF) on a Bruker REFLEX II instrument. Homogeneity was evaluated using analytical RP-HPLC (Vydac C4 silica column, $5 \mu \mathrm{m}, 250 \mathrm{~mm} \times 4 \mathrm{~mm}$ ).

Circular Dichroism. Measurements were performed on an Aviv 62A DS Circular Dichroism Spectrometer. Data were acquired in quartz $0.1 \mathrm{~cm}$ cuvettes with $5 \mathrm{sec}$ averaging times. Samples were prepared by dissolving purified peptides in PBS ( $\mathrm{pH}$ 7.0) to the appropriate peptide concentration as determined by tryptophan absorbance in $6 \mathrm{M}$ guanidine hydrochloride. ${ }^{\mathrm{S} 7}$ Thermal denaturations were monitored by ellipticity at $222 \mathrm{~nm}$ with a temperature step size of $3{ }^{\circ} \mathrm{C}$ and equilibration times of $3-8$ min at each temperature. Data were treated for folded and unfolded baselines and converted to fraction unfolded $\left(\mathrm{F}_{\text {unf }}\right)$ vs. temperature. Melting temperatures $\left(\mathrm{T}_{\mathrm{m}}\right)$ were obtained from the minimum of the first derivative of a $\mathrm{F}_{\text {unf }}$ vs. $1 / \mathrm{T}$ plot. ${ }^{\mathrm{S} 8}$ All melts were reversible, provided that the samples were not subjected to high temperatures for extended periods of time. Urea denaturations were performed at 25 ${ }^{\circ} \mathrm{C}$ with peptide concentrations ranging from $25 \mu \mathrm{M}$ to $160 \mu \mathrm{M}$. The free energy of unfolding at each urea concentration was determined based on a monomer-dimer equilibrium model ( $\left.F_{2} \leftrightarrow 2 U\right)$, with the expression $\Delta \mathrm{G}_{(\text {unf, urea })}=-\mathrm{RT} \ln \left[\left(2[\mathrm{P}]_{\text {total }} \mathrm{F}_{\text {unf }}^{2}\right) /\left(1-\mathrm{F}_{\text {unf }}\right)\right]$ where $[\mathrm{P}]_{\text {total }}$ is total monomer concentration, $\mathrm{F}_{\text {unf }}$ is the fraction unfolded, $\mathrm{R}$ is the gas constant, and $\mathrm{T}$ is temperature. ${ }^{\mathrm{S} 9}$ The unfolding energy in water could then be extrapolated using the equation $\Delta \mathrm{G}_{(\mathrm{unf}, \text { urea) }}=\Delta \mathrm{G}_{(\mathrm{unf}, \text { buffer) }}+m$ [urea].

Analytical Ultracentrifugation. Sedimentation studies were conducted on a Beckman XLA ultracentrifuge at $25{ }^{\circ} \mathrm{C}$. Peptide solutions in PBS were loaded into $1.2 \mathrm{~cm}$ cells and absorbance monitored at $280 \mathrm{~nm}$ or $231 \mathrm{~nm}$. Analysis was performed at several rotor speeds ranging from 24 to 36 krpm. At each speed, data were collected with a $0.001 \mathrm{~cm}$ step size every $4 \mathrm{hr}$ until three consecutive 
spectra were identical (typical equilibration times were $20-28 \mathrm{hr}$ ). The data were well described by an ideal single species model with a molecular weight consistent with the Base-L21A/pLEIM heterodimer. Non-linear regression was performed in accordance with the expression $c_{r}=c_{o} \exp \left[M(1-v \rho) \omega^{2}\left(r^{2}-\right.\right.$ $\left.\left.r_{o}^{2}\right) / 2 \mathrm{RT}\right]+$ base, where $c_{r}$ is the concentration (in absorbance units) at radial position $r, c_{o}$ is the concentration at an arbitrary reference position $r_{o}$ near the meniscus, $v$ is the partial specific volume, $\rho$ is the solvent density, $\omega$ is the rotor speed, $\mathrm{R}$ is the gas constant, $\mathrm{T}$ is the temperature, and base is a baseline absorbance correction to account for non-sedimenting species. ${ }^{\text {S10 }}$ Molecular weight estimates were obtained from the parameter $M$. Fits were judged to be adequate by randomness of residuals. A partial specific volume of $0.755 \mathrm{~mL} / \mathrm{g}$ was calculated based on amino acid composition, ${ }^{\mathrm{S} 11}$ and a solvent density of $1.004550 \mathrm{~g} / \mathrm{mL}$ was determined using an Anton Parr DMA 5000 density meter. The best sedimentation results were obtained at $80 \mu \mathrm{M}$ total peptide concentration. However, CD spectra at several concentrations ranging from $10 \mu \mathrm{M}$ to $140 \mu \mathrm{M}$ were identical, indicating that the aggregation state of Base-p1-L21A/pLEIM does not vary in this range.

Thiol Exchange Assay. Disulfide-bonded peptides were obtained by air oxidation at pH $8.5-9$ for $\sim 20$ hr, followed by RP-HPLC purification as above. Thiol exchange assays were performed in degassed PBS (pH 7.0) containing $1 \mathrm{mM}$ EDTA under inert atmosphere at room temperature. Following the appropriate reaction time, aliquots were quenched with glacial acetic acid (to $10 \%(\mathrm{v} / \mathrm{v})$ ) and analyzed immediately by RP-HPLC (C4 analytical column with water/acetonitrile/TFA mobile phases; the identity of each species was confirmed by co-elution with authentic standards). Several independent trials of each reaction produced identical results. Reactant/product ratios were invariant for reaction times ranging from $20 \mathrm{~min}$ to $3 \mathrm{hr}$.

\section{ACKNOWLEDGEMENTS}

$\mathrm{CD}$ and AU data were obtained at the University of Wisconsin - Madison Biophysics Instrumentation Facility, which is supported by the UW - Madison and grants BIR-9512577 (NSF) 
and S10 RR13790 (NIH). DNA synthesis and sequencing was performed at the UW - Madison

Biotechnology Center DNA Synthesis/Sequencing Facility.

\section{REFERENCES}

S1. Fowlkes, D., Adams, M., Fowler, V. \& Kay, B. Multipurpose vectors for peptide expression on the M13 viral surface. BioTechniques. 1992, 13, 422-427.

S2. Moitra, J., Szilak, L., Krylov, D. \&Vinson, C. Leucine is the most stabilizing aliphatic amino acid in the $\mathbf{d}$ position of a dimeric leucine zipped coiled coil. Biochemistry. 1997, 36, 12567-12573.

S3. Acharya, A., Ruvinov, S. B., Gal, J., Moll, J. R. \& Vinson, C. A heterodimerizing leucine coiled coil system for examining the specificity of a position interactions: Amino acids I, V, L, N, A, and K. Biochemistry. 2002, 41, 14122-14131.

S4. Wagschal, K., Tripet, B., Lavigne, P., Mant, C. \& Hodges, R. S. The role of position a in determining the stability and oligomerization state of $\alpha$-helical coiled coils: 20 amino acid stability coefficients in the hydrophobic core positions. Protein Sci. 1999, 8, 2312-2329.

S5. Kay, B. K., Winter, J. \& McCafferty, J., Eds. Phage display of peptides and proteins: A laboratory manual. Academic Press: New York, NY. (1996)

S6. Kay, B. K., Kasanov, J. \& Yamabhai, M. Screening phage-displayed combinatorial peptide libraries. Methods. 2001, 24, 240-246.

S7. Edelhoch, H. Spectroscopic determination of tryptophan and tyrosine in proteins. Biochemistry. 1967, 6, 1948-1959.

S8. Cantor, C. R. \& Schimmel, P. R. Biophysical chemistry part III: The behavior of biological macromolecules. W. H. Freeman and company: New York, NY. (1998) p1132.

S9. Pace, C. N. Determination and analysis of urea and guanidine hydrochloride denaturation curves. Methods Enzym. 1986, 131, 266-280.

S10. Schuster, T. M. \& Laue, T. M., Eds. Modern analytical ultracentrifugation. Birkhauser: Boston, MA. (1994).

S11. Harding, S. E., Rowe, A. J. \& Horton, J. S, Eds. Analytical ultracentrifugation in biochemistry and polymer science. The Royal Society of Chemistry: Cambridge, UK. (1992) pp97-103. 